\title{
Aplicações do Modified Early Warning Score pelo enfermeiro em hospitais
}

\author{
Application of the Modified Early Warning Score by Nurse in Hospitals \\ Aplicaciones de la Modified Early Warning Score por enfermeras en hospitales
}

Recebido: 07/01/2022 | Revisado: 170/01/2022 | Aceito: 20/01/2022 | Publicado: 22/01/2022

Luzia Cibele de Souza Maximiano

ORCID: https://orcid.org/0000-0001-8961-6239

Universidade do Estado do Rio Grande do Norte, Brasil

E-mail: luziacibele42@gmail.com

Maria Eduarda da Silva Araújo

ORCID: https://orcid.org/0000-0002-3616-2058

Universidade do Estado do Rio Grande do Norte, Brasil

E-mail: eduardasilva051420@gmail.com

Alcivan Nunes Vieira

ORCID: https://orcid.org/0000-0003-4222-6262

Universidade do Estado do Rio Grande do Norte, Brasil E-mail: alcivannunes@uern.br

Luana Adrielle Leal Dantas

ORCID: https://orcid.org/0000-0001-6747-3513

Universidade do Estado do Rio Grande do Norte, Brasil

E-mail: luanadantas904@gmail.com

Lucidio Clebeson de Oliveira

ORCID: https://orcid.org/0000-0002-2033-7546

Universidade do Estado do Rio Grande do Norte, Brasil

E-mail: lucidioclebeson@hotmail.com

Helder Barbosa Rodrigues

ORCID: https://orcid.org/0000-0002-2493-2211

Universidade Federal Rural do Semi-Árido, Brasil

E-mail: hellder.b.rodrigues@gmail.com

\begin{abstract}
Resumo
Objetivo: identificar como o Modified Early Warning Score (MEWS) tem sido aplicado na prática clínica hospitalar pelo enfermeiro. Método: trata-se de uma revisão integrativa da literatura; para a elaboração da questão norteadora utilizou-se a estratégia PICO conforme recomendação do Reporting Items for Systematic Reviews and Meta-Analyses (PRISMA): O nível de evidência dos estudos selecionados foi baseado no Oxford Centre for Evidence-based Medicine. Resultados: identificaram-se 24 artigos nas seguintes fontes: PUBMED, SCIELO, LILACS, SCOPUS e WEB OF SCIENCE; os níveis de evidência foram: 7 artigos classificados com evidência 1A; 12 artigos classificados com 2A; 1 artigo classificado como 2A; 2 artigos $2 \mathrm{C}$ e 2 artigos classificados como $3 \mathrm{~A}$. O MEWS é aplicado como escore preditor de deterioração clínica das funções vitais, na identificação do risco para ocorrência de Parada Cardiorrespiratória e no estabelecimento de critérios para admissão na UTI. Conclusão: contatou-se que a utilização do MEWS na prática clínica hospitalar pelo enfermeiro objetiva a avaliação do estado de saúde do paciente, identificando sinais de deterioração grave das funções vitais. Ampara a adoção dos princípios de Segurança do Paciente além de favorecer a comunicação entre a equipe agiliza intervenções do Time de Resposta Rápida.
\end{abstract}

Palavras-chave: Unidade de terapia intensiva; Escore de alerta precoce; Cuidados críticos.

\begin{abstract}
Objective: to identify how the Modified Early Warning Score (MEWS) has been applied in hospital clinical practice by nurses. Method: it is an integrative literature review; for the elaboration of the guiding question, the PICO strategy was used, as recommended by the Reporting Items for Systematic Reviews and Meta-Analyses (PRISMA): The level of evidence of the selected studies was based on the Oxford Center for Evidence-based Medicine. Results: 24 articles were identified in the following sources: PUBMED, SCIELO, LILACS, SCOPUS and WEB OF SCIENCE; the levels of evidence were: 7 articles classified as $1 \mathrm{~A}$ evidence; 12 articles rated 2A; 1 article rated 2A; $22 \mathrm{C}$ articles and 2 articles classified as 3A. The MEWS is applied as a predictor score for clinical deterioration of vital functions, in identifying the risk for the occurrence of cardiopulmonary arrest and establishing criteria for admission to the ICU. Conclusion: it was found that the use of MEWS in hospital clinical practice by nurses aims to assess the patient's health status, identifying signs of serious deterioration of vital functions. It supports the adoption of the principles of Patient Safety, in addition to favoring communication between the team, streamlines interventions by the Rapid Response Team.
\end{abstract}

Keywords: Intensive care Unit; Early warning score; Critical care. 


\begin{abstract}
Resumen
Objetivo: Identificar cómo las enfermeras han aplicado la puntuación de alerta temprana modificada (MEWS) en la práctica clínica hospitalaria. Método: se trata de una revisión integradora de la literatura; Se utilizó la estrategia PICO para preparar la pregunta guía, según lo recomendado por Reporting Items for Systematic Reviews and MetaAnalyzes (PRISMA): El nivel de evidencia de los estudios seleccionados se basó en el Oxford Center for Evidencebased Medicine. Resultados: Se identificaron 24 artículos de las siguientes fuentes: PUBMED, SCIELO, LILACS, SCOPUS y WEB OF SCIENCE; los niveles de evidencia fueron: 7 artículos clasificados como evidencia 1A; 12 artículos clasificados 2A; 1 artículo clasificado 2A; 2 artículos 2C y 2 artículos clasificados como 3A. La MEWS se aplica como score predictor de deterioro clínico de las funciones vitales, en la identificación del riesgo de ocurrencia de parada cardiorrespiratoria y en el establecimiento de criterios de ingreso en UCI. Conclusión: se encontró que el uso de MEWS en la práctica clínica hospitalaria por parte del personal de enfermería tiene como objetivo evaluar el estado de salud del paciente, identificando signos de deterioro grave de las funciones vitales. Apoya la adopción de los principios de Seguridad del Paciente, además de favorecer la comunicación entre el equipo, agiliza las intervenciones del Equipo de Respuesta Rápida.
\end{abstract}

Palabras clave: Unidad de cuidados intensivos; Puntuación de alerta temprana; Cuidado crítico.

\title{
1. Introdução
}

Caracterizam-se os hospitais como instituições cuja função primordial é assegurar a realização de intervenções voltadas para a cura ou reabilitação do paciente. Sabe-se que com o crescimento da procura por uma assistência progressivamente mais complexa, a assistência vem sendo organizada por especialidades e em níveis de complexidade, conforme a necessidade do paciente. Destaca-se que esta forma de organização é essencial para concretizar os princípios de Segurança do Paciente e de um atendimento orientado por uma atenção eficaz, efetiva e interprofissional (Cipriano, Salgado, Oliveira \& Aguiar, 2018).

Identificou-se que a maioria dos pacientes criticamente enfermos que chega às unidades de emergência podem apresentar sinais de gravidade clínica que, se forem detectados precocemente, evidenciam a deterioração do seu estado clínico sendo que aproximadamente $80 \%$ destes sinais podem ser identificados 24 horas antes desse agravamento (Rocha, Neves \& Viegas, 2016). Torna-se relevante esta identificação precoce, uma vez que a taxa de mortalidade nas Unidades de Terapia Intensiva (UTI) brasileiras em 2015 era de 12,24\%, seguindo por uma queda no ano posterior $(11,82 \%)$ e alcançando $10,76 \%$ em 2019 (Associação de Medicina Intensiva Brasileira, 2020).

Destaca-se que a avaliação realizada pelo profissional de saúde, através da observação e monitorização contínua dos pacientes, muitas vezes define e antecipa as condutas a serem adotadas. Infere-se que esta avaliação pode ser realizada com mais precisão e objetividade se forem utilizados escores prognósticos já validados cientificamente; pois, evidenciou-se que a utilização desses instrumentos uniformiza e aprimora a avaliação clínica desenvolvida pelos profissionais (Bekhit, Algameel, \& Eldash, 2014).

Detalha-se que os escores preditores de gravidade clínica são cada vez mais utilizados nos ambientes hospitalares objetivando a formulação de prognósticos, estabelecimento de prioridades, na caracterização e evolução da gravidade da doença. Ressalta-se que outros propósitos para a sua aplicação incluem avaliar o grau de disfunção orgânica e o resultado das intervenções realizadas nas unidades assistenciais (Bekhit et al., 2014).

Observa-se que é imprescindível conhecer e identificar sinais de alerta da deterioração clínica em pacientes graves. Identificando-se que para esta finalidade vários escores que englobam diferentes parâmetros foram elaborados, dentre eles destaca-se o Escore de Alerta Precoce (Early Warning Scores - EWS) e a sua versão modificada, o Modified Early Warning Score - MEWS (Morales, 2016).

Salienta-se que o MEWS pode ser aplicado pela equipe de enfermagem quando esses profissionais verificam os sinais vitais do paciente. Esta avaliação consiste na atribuição valores de 0 a 3 para os seguintes parâmetros: frequência respiratória, frequência cardíaca, pressão arterial sistólica, nível de consciência, temperatura. A soma desses valores representa o MEWS 
que é capaz de indicar se há deterioração das funções vitais, consequentemente, evidencia aqueles pacientes que necessitam de maior atenção por parte da equipe multiprofissional (Morales, 2016).

Destaca-se neste sentido, que a aplicação do MEWS pelo enfermeiro pode apresentar-se como uma ferramenta capaz de identificar precocemente a gravidade clínica, além de favorecer o alerta aos outros membros da equipe multiprofissional, permitindo uma intervenção efetiva que seja capaz de abordar a piora clínica, prevenindo a ocorrência de eventos graves com prognósticos desfavoráveis. O MEWS apresenta-se como um instrumento que pode ser aplicado à beira do leito, com custo efetividade compatível com a estrutura de vários serviços e unidades assistenciais (Montenegro, 2017).

Ressalta-se que com o início da pandemia global pelo Sars-Cov-2, a procura por assistência hospitalar em casos graves aumentou a demanda nos serviços de urgência e emergência, necessitando assim de uma avaliação clínica precisa para uma intervenção precoce e imediata. Contatou-se que segundo a OMS, $80 \%$ dos pacientes com COVID-19 apresentam sintomas leves e sem complicações, $15 \%$ evoluem para hospitalização que necessita de oxigenoterapia e 5\% precisam ser atendidos em unidade de terapia intensiva (UTI) (World Health Organization, 2020). Sugere-se que a depender da velocidade de propagação do vírus na população, os sistemas de saúde podem sofrer forte pressão decorrente da demanda extra gerada pela COVID-19 (Noronha, Guedes, Turra, Andrade, Botega \& Nogueira, 2020).

Orienta-se em estudo que os pacientes hospitalizados com insuficiência respiratória sejam monitoramentos de maneira regular com verificação dos sinais vitais e, sempre que possível, utilização de escores de alerta que facilitam o reconhecimento precoce e a intensificação do tratamento do paciente em deterioração (BRASIL, 2020).

Este estudo tem como objetivo identificar como o Modified Early Warning Score (MEWS) tem sido aplicado na prática clínica hospitalar pelo enfermeiro.

\section{Metodologia}

Trata-se de um estudo bibliográfico, descritivo, tipo Revisão Integrativa da Literatura organizada nas seguintes etapas: elaboração do protocolo da revisão; coleta dos dados com inclusão de publicações que trouxeram contribuições e respostas pertinentes à questão de pesquisa; extração das informações contidas no estudo; análise e interpretação dos dados; e, por último, apresentação e discussão da revisão (Botelho, Cunha \& Macedo, 2011).

Elaborou-se a questão norteadora, utilizando-se da estratégia PICO conforme recomendação do Reporting Items for Systematic Reviews and Meta-Analyses (PRISMA): População: pacientes internados em unidades assistenciais hospitalares, Intervenção: aplicação de escore preditor de gravidade clínica, Comparação: com pacientes que não foram submetidos a avaliação com escore prognóstico, Outcomes (desfecho): identificação precoce da deterioração clínica.

Investigou-se as publicações científicas indexadas nas seguintes fontes de pesquisa: LILACS, SCIELO, PUBMED, SCOPUS e WEB OF SCIENCE. Realizou-se a coleta de dados em Maio de 2020 com uso de descritores selecionados nos Descritores em Ciências da Saúde (DECs): 1\#Unidade de terapia intensiva/Intensive Care Units; 2\#Escore de alerta precoce/Early warning score; 3\#Cuidados críticos/Critical Care. Incluiram-se os artigos completos de forma livre via plataforma de busca do Periódicos CAPES, disponíveis em qualquer idioma e publicados entre os anos de 2016 a 2020. Excluiram-se as publicações em formato de cartas ao editor, resumos, editoriais, capítulos de livros, teses e dissertações ou que não abordassem o tema da pesquisa. Utilizou-se como cruzamentos nas buscas: (intensive care unit AND eartly warning score); (early warning score AND critical care); (intensive care unit AND early warning score AND critical care).

Iniciou-se a seleção das publicações por meio da retirada das duplicatas, pela leitura dos títulos e dos resumos dos trabalhos. Retirou-se posteriormente os estudos não disponíveis na íntegra, e os que possuíam dados insuficientes para responderem à questão de pesquisa, obtendo-se o total de 24 publicações. Classificou-se o nível de evidência dos estudos selecionados com base no Oxford Centre for Evidence-based Medicine (2009) e, ao todo, foram classificados como: 7 artigos 
classificados como 1A (Revisão sistemática de estudos de coorte - contemporânea ou prospectiva); 12 artigos classificados com 2A (revisão sistemática de coortes históricas -retrospectivas); 1 artigo classificado como 1A e 2A por conter abordagem retrospectiva e prospectiva; 2 artigos $2 \mathrm{C}$ (observação de resultados terapêuticos -outcomes research) e, por fim, 2 artigos classificados como 3A (revisão sistemática de estudos caso-controle).

A Figura 1 representa o fluxograma da seleção de artigos para a revisão.

Figura 1: Fluxograma de seleção dos artigos para a revisão integrativa adaptado do Preferred Reporting Items for Systematic Reviews and MetaAnalyses (PRISMA 2009). Mossoró (RN), Brasil, 2020.

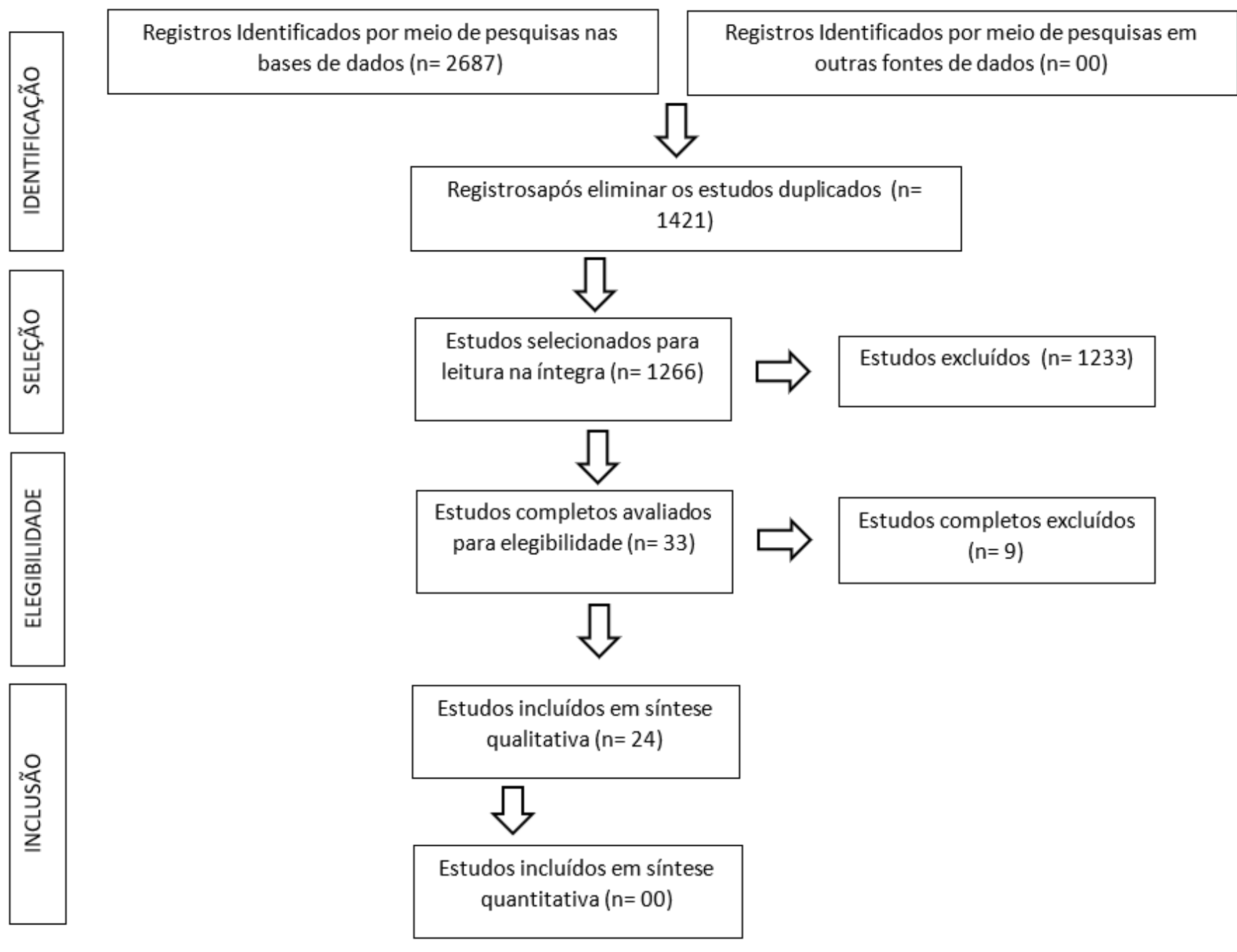

Fonte: Autores (2020).

\section{Resultados}

Identificou-se que as publicações selecionadas estão no período de 2016 a 2020. Nota-se que quanto ao idioma, três foram escritos em português, e os demais em inglês. Revelou-se ainda que os estudos tiveram os seguintes países de origem: Brasil, Turquia, Tailândia, Noruega, China, Estados Unidos, Itália, Alemanha, Canadá, Coreia do Sul, Japão, Grécia, Etiópia, Uganda e Reino Unido. Observou-se que, no Brasil, o quantitativo de pesquisas da temática é limitado a três estudos, dos quais 1 é um estudo transversal retrospectivo, outro um estudo analítico preditivo e o terceiro sendo um estudo de caso-controle. Verificaram-se poucas publicações vinculadas à revistas de enfermagem. 
Observou-se em resposta ao questionamento da presente revisão, que o MEWS utilizado na prática clínica hospitalar possui bons valores preditivos para identificação precoce da Parada Cardíaca, risco de ocorrência de óbito e estabelecer critérios para admissão na UTI, além de modificar-se para atender às demandas institucionais de cada local.

Efetuou-se a apresentação dos artigos quanto as variáveis: autor e ano de publicação, periódico/título, objetivo, metodologia e conclusão, as quais estão caracterizadas no Quadro 1.

Quadro 1. Síntese da distribuição das publicações cientifica da revisão Integrativa segundo ano, autor, periódico, base de dados, título, objetivo, metodologia e conclusão. Mossoró (RN), Brasil, 2020.

\begin{tabular}{|c|c|c|c|c|c|}
\hline Autor/ano & $\begin{array}{l}\text { Periódico/base } \\
\text { de dados }\end{array}$ & Título & Objetivo & Metodologia & Conclusão \\
\hline $\begin{array}{l}\text { Gok, R. G. Y. } \\
2019\end{array}$ & $\begin{array}{l}\text { International } \\
\text { Emergency } \\
\text { Nursing/ } \\
\text { PUBMED }\end{array}$ & $\begin{array}{l}\text { Assessing prognosis with } \\
\text { modified early warning } \\
\text { score, rapid emergency } \\
\text { medicine score and } \\
\text { worthing physiological } \\
\text { scoring system in patients } \\
\text { admitted to intensive care } \\
\text { unit from emergency } \\
\text { department }\end{array}$ & $\begin{array}{l}\text { Examinar a eficácia e a } \\
\text { confiabilidade do } \\
\text { MEWS, REMS e WPS } \\
\text { como sistemas de } \\
\text { pontuação para predição } \\
\text { do prognóstico e taxa de } \\
\text { mortalidade de pacientes } \\
\text { críticos programados } \\
\text { para internação na UTI } \\
\text { entre os pacientes do } \\
\text { departamento de } \\
\text { emergência }\end{array}$ & $\begin{array}{l}\text { Estudo } \\
\text { retrospectivo } \\
\text { (evidência 2A) }\end{array}$ & $\begin{array}{l}\text { Houve uma correlação mais } \\
\text { significativa entre o escore WPS } \\
\text { e mortalidade/ tempo de } \\
\text { permanência na UTI em } \\
\text { comparação com outros escores. }\end{array}$ \\
\hline $\begin{array}{l}\text { Khwannimit, } \\
\text { B. } 2019\end{array}$ & $\begin{array}{l}\text { Heart \& } \\
\text { Lung/PUBME } \\
\text { D }\end{array}$ & $\begin{array}{l}\text { Comparison of the } \\
\text { accuracy of three early } \\
\text { warning scores with } \\
\text { SOFA score for } \\
\text { predicting mortality in } \\
\text { adult sepsis and septic } \\
\text { shock patients admitted } \\
\text { to intensive care unit }\end{array}$ & $\begin{array}{l}\text { Comparar a precisão } \\
\text { MEWS, NEWS) e SOS, } \\
\text { com o qSOFA e escores } \\
\text { SOFA para prever } \\
\text { resultados em pacientes } \\
\text { com sepse. }\end{array}$ & $\begin{array}{l}\text { Estudo } \\
\text { retrospectivo } \\
\text { (evidência 2A) }\end{array}$ & $\begin{array}{l}\text { O SOS é quase tão bom quanto o } \\
\text { escore SOFA para prever a } \\
\text { mortalidade entre pacientes com } \\
\text { sepse na UTI. O MEWS é outra } \\
\text { ferramenta alternativa a ser usada } \\
\text { para estratificação de risco e } \\
\text { sepse na UTI }\end{array}$ \\
\hline $\begin{array}{l}\text { Stafseth, S. K. } \\
2016\end{array}$ & $\begin{array}{l}\text { ScienceDirect/ } \\
\text { PUBMED }\end{array}$ & $\begin{array}{l}\text { The experiences of nurses } \\
\text { implementing the } \\
\text { Modified Early Warning } \\
\text { Score and a 24-hur on- } \\
\text { call Mobile Intensive } \\
\text { Care Nurse: An } \\
\text { exploratory study }\end{array}$ & $\begin{array}{l}\text { Explorar experiências de } \\
\text { enfermeiros } \\
\text { implementando e usando } \\
\text { o MEWS }\end{array}$ & $\begin{array}{l}\text { Estudo } \\
\text { qualitativo } \\
\text { exploratório, } \\
\text { utilizando } \\
\text { entrevistas } \\
\text { semiestruturad } \\
\text { as de grupos } \\
\text { focais } \\
\text { (evidência 2C) }\end{array}$ & $\begin{array}{l}\text { Os enfermeiros aumentaram a } \\
\text { confiança no reconhecimento de } \\
\text { pacientes em deterioração e no } \\
\text { gerenciamento de tais situações. }\end{array}$ \\
\hline Hu, H. 2020 & $\begin{array}{l}\text { Society for } \\
\text { Academic } \\
\text { Emergency } \\
\text { Medicine/PUB } \\
\text { MED }\end{array}$ & $\begin{array}{l}\text { Comparing Rapid } \\
\text { Scoring Systems in } \\
\text { Mortality Prediction of } \\
\text { Critically Ill Patients } \\
\text { With Novel Coronavirus } \\
\text { Disease }\end{array}$ & $\begin{array}{l}\text { Avaliar o desempenho do } \\
\text { sistema de pontuação } \\
\text { rápida na admissão de } \\
\text { pacientes com COVID19 }\end{array}$ & $\begin{array}{l}\text { Revisão } \\
\text { retrospectiva } \\
\text { de relatórios de } \\
\text { assistência ao } \\
\text { paciente de } \\
\text { uma equipe } \\
\text { médica de } \\
\text { emergência de } \\
\text { um hospital } \\
\text { chinês. } \\
\text { (evidência 2A) }\end{array}$ & $\begin{array}{l}\text { O MEWS demonstra valores } \\
\text { preditivos aceitáveis para a } \\
\text { mortalidade hospitalar de } \\
\text { pacientes gravemente enfermos } \\
\text { com COVID-19. }\end{array}$ \\
\hline $\begin{array}{l}\text { Salottolo, K. } \\
2017\end{array}$ & $\begin{array}{l}\text { BMJ } \\
\text { Open/PUBME } \\
\text { D }\end{array}$ & $\begin{array}{l}\text { A retrospective cohort } \\
\text { study of the utility of the } \\
\text { modified early warning } \\
\text { score for interfacility }\end{array}$ & $\begin{array}{l}\text { Determinar se o MEWS } \\
\text { pode ser usado para } \\
\text { prever resultados em } \\
\text { pacientes traumatizados }\end{array}$ & $\begin{array}{l}\text { Estudo } \\
\text { retrospectivo } \\
\text { de pacientes } \\
\text { traumatizados }\end{array}$ & $\begin{array}{l}\text { MEWS } \geq 4 \text { pode ser usada com } \\
\text { facilidade para prever a gravidade } \\
\text { da lesão, mortalidade, transporte e } \\
\text { uso de recursos da UTI. }\end{array}$ \\
\hline
\end{tabular}




\begin{tabular}{|c|c|c|c|c|c|}
\hline & & $\begin{array}{l}\text { transfer of patients with } \\
\text { traumatic injury }\end{array}$ & $\begin{array}{l}\text { exigindo transferência } \\
\text { para níveis mais altos de } \\
\text { atendimento. }\end{array}$ & $\begin{array}{l}\text { transferidos } \\
\text { para um nível } \\
\text { II de trauma de } \\
2013 \text { a } 2014 . \\
\text { (evidência } 2 A \text { ) }\end{array}$ & \\
\hline Xie, X. 2018 & $\begin{array}{l}\text { BMJ } \\
\text { Open/PUBME } \\
\text { D }\end{array}$ & $\begin{array}{l}\text { Prognostic value of } \\
\text { Modified Early Warning } \\
\text { Score generated in a } \\
\text { Chinese emergency } \\
\text { department: a prospective } \\
\text { cohort study }\end{array}$ & $\begin{array}{l}\text { Validar o desempenho do } \\
\text { MEWS em um } \\
\text { departamento chinês de } \\
\text { emergência e determinar } \\
\text { o melhor ponto de corte } \\
\text { para a previsão de } \\
\text { mortalidade hospitalar. }\end{array}$ & $\begin{array}{l}\text { Coorte } \\
\text { observacional } \\
\text { prospectivo, } \\
\text { centrada em } \\
\text { um único } \\
\text { estudo } \\
\text { (evidência 1A) }\end{array}$ & $\begin{array}{l}\text { Apoiam o uso de MEWS para } \\
\text { predição intrahospitalar de } \\
\text { mortalidade em pacientes que } \\
\text { foram triados }\end{array}$ \\
\hline $\begin{array}{l}\text { Tirotta, D. } \\
2017\end{array}$ & $\begin{array}{l}\text { International } \\
\text { Journal of } \\
\text { Medicine/PUB } \\
\text { MED }\end{array}$ & $\begin{array}{l}\text { Evaluation of the } \\
\text { threshold value for the } \\
\text { modified early warning } \\
\text { score (MEWS) in } \\
\text { medical septic patients: a } \\
\text { secondary analysis of an } \\
\text { Italian multicentric } \\
\text { prospective cohort } \\
\text { (SNOOPII study) }\end{array}$ & $\begin{array}{l}\text { Explorar a capacidade do } \\
\text { MEWS para prever a } \\
\text { mortalidade hospitalar } \\
\text { em pacientes sépticos } \\
\text { admitidos nas } \\
\text { enfermarias. }\end{array}$ & $\begin{array}{l}\text { Análise } \\
\text { secundária de } \\
\text { um estudo } \\
\text { prospectivo } \\
\text { multicêntrico. } \\
\text { (Evidência 1A) }\end{array}$ & $\begin{array}{l}\text { Os achados não parecem apoiar o } \\
\text { uso de MEWS para prever o risco } \\
\text { de mortalidade hospitalar de } \\
\text { sepse em enfermarias. }\end{array}$ \\
\hline $\begin{array}{l}\text { Heller, A. R. } \\
2020\end{array}$ & $\begin{array}{l}\text { Annals of } \\
\text { Surgery/PUB } \\
\text { MED }\end{array}$ & $\begin{array}{l}\text { Detection of } \\
\text { Deteriorating Patients on } \\
\text { Surgical Wards Outside } \\
\text { the ICU by an Automated } \\
\text { MEWS-Based Early } \\
\text { Warning System With } \\
\text { Paging Functionality }\end{array}$ & $\begin{array}{l}\text { Analisar o efeito da } \\
\text { introdução do MEWS em } \\
2 \text { enfermarias que } \\
\text { hospedam pacientes em } \\
\text { recuperação de } \\
\text { intervenções cirúrgicas } \\
\text { altamente complexas. }\end{array}$ & $\begin{array}{l}\text { Estudo } \\
\text { retrospectivo } \\
\text { (evidência 2A) }\end{array}$ & $\begin{array}{l}\text { O início de uma detecção de } \\
\text { deterioração de pacientes em } \\
\text { enfermarias cirúrgicas fora da } \\
\text { UTI pode ser aprimorada com a } \\
\text { introdução do MEWS. }\end{array}$ \\
\hline $\begin{array}{l}\text { Kruisselbrink } \\
\text {, R.2016 }\end{array}$ & $\begin{array}{l}\text { PLOS } \\
\text { ONE/PUBME } \\
\text { D }\end{array}$ & $\begin{array}{l}\text { Modified EarlyWarning } \\
\text { Score (MEWS) Identifies } \\
\text { Critical Illness among } \\
\text { Ward Patients in a } \\
\text { Resource Restricted } \\
\text { Setting in Kampala, } \\
\text { Uganda: A Prospective } \\
\text { Observational Study }\end{array}$ & $\begin{array}{l}\text { Determinar o prevalência } \\
\text { de doenças críticas nas } \\
\text { enfermarias, conforme } \\
\text { definido pelo MEWS, } \\
\text { bem como avaliar o } \\
\text { MEWS como preditor de } \\
\text { morte e para descrever } \\
\text { fatores de risco } \\
\text { adicionais para } \\
\text { mortalidade. }\end{array}$ & $\begin{array}{l}\text { Estudo } \\
\text { observacional } \\
\text { prospectivo. } \\
\text { (Evidência 1A) }\end{array}$ & $\begin{array}{l}\text { O MEWS pode fornecer uma } \\
\text { ferramenta de triagem útil para } \\
\text { identificar pacientes com maior } \\
\text { risco de morte. Pesquisas futuras } \\
\text { devem incluir o aprimoramento } \\
\text { do MEWS para configurações de } \\
\text { poucos recursos, } \\
\text { desenvolvimento e intervenções } \\
\text { apropriadas para pacientes } \\
\text { identificados como de alto risco } \\
\text { de morte nas pontuações de alerta } \\
\text { precoce. }\end{array}$ \\
\hline $\begin{array}{l}\text { Lee, J. R. } \\
2020\end{array}$ & $\begin{array}{l}\text { The Korean } \\
\text { Association of } \\
\text { Internal } \\
\text { Medicine/PUB } \\
\text { MED }\end{array}$ & $\begin{array}{l}\text { Derivation and validation } \\
\text { of modified early } \\
\text { warning score plus } \\
\text { SpO2/FiO2 score for } \\
\text { predicting acute } \\
\text { deterioration of patients } \\
\text { with } \\
\text { hematologicalmalignanci } \\
\text { es }\end{array}$ & $\begin{array}{l}\text { Avaliar o MEWS para } \\
\text { prever internações em } \\
\text { UTI e mortalidade } \\
\text { hospitalar entre pacientes } \\
\text { em risco, pacientes com } \\
\text { neoplasias hematológicas } \\
\text { e desenvolver um MEWS } \\
\text { otimizado. }\end{array}$ & $\begin{array}{l}\text { Estudo } \\
\text { retrospectivo } \\
\text { com pacientes } \\
\text { com } \\
\text { malignidades } \\
\text { hematológicas } \\
\text { administradas } \\
\text { por uma equipe } \\
\text { de emergência } \\
\text { médica } \\
\text { (evidência 2A) }\end{array}$ & $\begin{array}{l}\text { Comparado ao MEWS } \\
\text { tradicional, o MEWS_SF pode } \\
\text { ser uma ferramenta útil que pode } \\
\text { ser usada na enfermaria geral para } \\
\text { identificar pacientes em } \\
\text { deterioração com malignidades } \\
\text { hematológicas. }\end{array}$ \\
\hline $\begin{array}{l}\text { Akgun, F. S. } \\
2018\end{array}$ & $\begin{array}{l}\text { Nigerian } \\
\text { journal of } \\
\text { clinical } \\
\text { practice/PUB } \\
\text { MED }\end{array}$ & $\begin{array}{l}\text { The prognastic } \\
\text { efficiencies of modified } \\
\text { early warning score and } \\
\text { mainz emergency } \\
\text { evaluation score for } \\
\text { emergency department }\end{array}$ & $\begin{array}{l}\text { Avaliar a eficiência do } \\
\text { MEWS e MEES na } \\
\text { avaliação da gravidade da } \\
\text { doença e na previsão } \\
\text { prognóstica a médio } \\
\text { prazo dos pacientes } \\
\text { hospitalizados após a }\end{array}$ & $\begin{array}{l}\text { Estudo } \\
\text { retrospectivo } \\
\text { (evidência 2A) }\end{array}$ & $\begin{array}{l}\text { A avaliação do MEWS é uma } \\
\text { ferramenta eficaz e confiável para } \\
\text { prever desfechos e hospitalização } \\
\text { de pacientes. Os valores MEES, } \\
\text { por outro lado, não são } \\
\text { convenientes para usar no }\end{array}$ \\
\hline
\end{tabular}




\begin{tabular}{|c|c|c|c|c|c|}
\hline & & patients & emergência. & & departamento de emergência. \\
\hline $\begin{array}{l}\text { Nishijima, I. } \\
2016\end{array}$ & $\begin{array}{l}\text { Journal of } \\
\text { Intensive } \\
\text { Care/PUBME } \\
\text { D }\end{array}$ & $\begin{array}{l}\text { Use of a modified early } \\
\text { warning score system to } \\
\text { reduce the rate of in- } \\
\text { hospital cardiac arrest }\end{array}$ & $\begin{array}{l}\text { Detectar anormalidades } \\
\text { precoces classificando } \\
\text { sinais vitais }\end{array}$ & $\begin{array}{l}\text { Estudo } \\
\text { retrospectivo } \\
\text { (evidência 2A) }\end{array}$ & $\begin{array}{l}\text { As paradas cardíacas diminuíram } \\
\text { devido à introdução do sistema, } \\
\text { pois o sistema permite } \\
\text { intervenções precoces para os } \\
\text { pacientes que têm a possibilidade } \\
\text { de deterioração aguda. }\end{array}$ \\
\hline $\begin{array}{l}\text { Zografakis- } \\
\text { Sfakianakis, } \\
\text { M. } 2018\end{array}$ & $\begin{array}{l}\text { Int J Nurs } \\
\text { Pract/PUBME } \\
\text { D }\end{array}$ & $\begin{array}{l}\text { The value of the } \\
\text { Modified Early Warning } \\
\text { Score for unplanned } \\
\text { Intensive Care Unit } \\
\text { admissions of patients } \\
\text { treated in hospital general } \\
\text { wards }\end{array}$ & $\begin{array}{l}\text { Determinar o valor da } \\
\text { pontuação de aviso } \\
\text { prévio do MEWS para a } \\
\text { ala geral e seu potencial } \\
\text { uso como ferramenta de } \\
\text { alarme para os } \\
\text { enfermeiros da } \\
\text { enfermaria. }\end{array}$ & $\begin{array}{l}\text { Estudo } \\
\text { observacional } \\
\text { prospectivo- } \\
\text { retrospectivo } \\
\text { combinado } \\
\text { com } \\
153 \text { pacientes } \\
\text { (Evidência 1A } \\
\text { e 2A) }\end{array}$ & $\begin{array}{l}\text { O MEWS é um forte indicador do } \\
\text { resultado e pode ser usado como } \\
\text { uma ferramenta de } \\
\text { monitoramento por mortes } \\
\text { potencialmente evitáveis e } \\
\text { internações não planejadas na } \\
\text { UTI. }\end{array}$ \\
\hline $\begin{array}{l}\text { Olino, L. } \\
2019\end{array}$ & $\begin{array}{l}\text { Rev Gaúcha de } \\
\text { Enf/SCIELO }\end{array}$ & $\begin{array}{l}\text { Comunicação efetiva } \\
\text { para a segurança do } \\
\text { paciente: nota de } \\
\text { transferência e Modified } \\
\text { Early Warning Score }\end{array}$ & $\begin{array}{l}\text { Analisar o registro da } \\
\text { Nota de Transferência e a } \\
\text { emissão do MEWS } \\
\text { realizados pelo } \\
\text { enfermeiro Em pacientes } \\
\text { adultos transferidos do } \\
\text { Serviço de Emergência } \\
\text { como estratégia de } \\
\text { comunicação efetiva para } \\
\text { a segurança do paciente. }\end{array}$ & $\begin{array}{l}\text { Estudo } \\
\text { transversal } \\
\text { retrospectivo } \\
\text { (evidência 2A) }\end{array}$ & $\begin{array}{l}\text { A NT e o MEWS estão inseridos } \\
\text { no trabalho do enfermeiro, no } \\
\text { entanto, são necessárias ações } \\
\text { com vistas a qualificar a } \\
\text { segurança do paciente, } \\
\text { melhorando a comunicação } \\
\text { efetiva e, por conseguinte, } \\
\text { diminuindo a possibilidade de } \\
\text { ocorrências de eventos adversos. }\end{array}$ \\
\hline $\begin{array}{l}\text { Montenegro, } \\
\text { S.M.S.L 2017 }\end{array}$ & $\begin{array}{l}\text { REBEn/SCIEL } \\
\mathrm{O}\end{array}$ & $\begin{array}{l}\text { Avaliação do } \\
\text { desempenho do MEWS } \\
\text { em hospital público } \\
\text { brasileiro }\end{array}$ & $\begin{array}{l}\text { Avaliar o desempenho do } \\
\text { MEWS em uma } \\
\text { enfermaria de pacientes } \\
\text { em deterioração clínica. }\end{array}$ & $\begin{array}{l}\text { Estudo } \\
\text { analítico, } \\
\text { quantitativo e } \\
\text { preditivo. } \\
\text { (Evidência 1A) }\end{array}$ & $\begin{array}{l}\text { Mews mensura adequadamente a } \\
\text { ocorrência de eventos graves em } \\
\text { pacientes hospitalizados em } \\
\text { enfermaria de um hospital público } \\
\text { brasileiro. Mews } \geq 4 \text { parece ser o } \\
\text { ponto de corte mais adequado } \\
\text { para predição destes eventos. }\end{array}$ \\
\hline $\begin{array}{l}\text { Alexandre, } \\
\text { A.R } 2019\end{array}$ & $\begin{array}{l}\text { Revista da } \\
\text { Sociedade } \\
\text { portuguesa de } \\
\text { medicina } \\
\text { intensiva/SCIE } \\
\text { LO }\end{array}$ & $\begin{array}{l}\text { Modified Early Warning } \\
\text { Score como Preditor de } \\
\text { Readmissões Precoces } \\
\text { Numa Unidade de } \\
\text { Cuidados Intensivos: Um } \\
\text { Estudo de Caso-Controle }\end{array}$ & $\begin{array}{l}\text { Testar o MEWS como } \\
\text { preditor de readmissões } \\
\text { precoces em UTI quando } \\
\text { calculado no momento da } \\
\text { alta destas unidades. }\end{array}$ & $\begin{array}{l}\text { Estudo de } \\
\text { caso-controle } \\
\text { (evidência 3A) }\end{array}$ & $\begin{array}{l}\text { O MEWS é um score clínico de } \\
\text { fácil aplicação, podendo } \\
\text { contribuir para aumentar a } \\
\text { segurança no momento da alta de } \\
\text { doentes internados em UTI. }\end{array}$ \\
\hline $\begin{array}{l}\text { Moore, J. } \\
2019\end{array}$ & $\begin{array}{l}\text { BMJ } \\
\text { Open/SCOPUS }\end{array}$ & $\begin{array}{l}\text { Introduction of a } \\
\text { modified obstetric early } \\
\text { warning system ( } \\
\text { MOEWS) at an Ethiopian } \\
\text { referral hospital: a } \\
\text { feasibility assessment }\end{array}$ & $\begin{array}{l}\text { Introduzir um sistema } \\
\text { obstétrico de alerta } \\
\text { precoce modificado } \\
\text { (MOEWS) avaliar sua } \\
\text { viabilidade e potencial } \\
\text { impacto na melhoria do } \\
\text { monitoramento pós- } \\
\text { operatório e identificação } \\
\text { precoce de deterioração } \\
\text { de pacientes }\end{array}$ & $\begin{array}{l}\text { Revisão } \\
\text { retrospectiva } \\
\text { trifásica de } \\
\text { realizada } \\
\text { durante um } \\
\text { período de } 1 \\
\text { ano (evidência } \\
\text { 2A) }\end{array}$ & $\begin{array}{l}\text { Com modificações para se } \\
\text { adequar ao ambiente e às } \\
\text { condições envolvimento clínico, } \\
\text { juntamente com treinamento } \\
\text { regular, a pontuação de alerta } \\
\text { precoce é uma ferramenta viável } \\
\text { e aceitável para lidar com as } \\
\text { demandas únicas enfrentadas em } \\
\text { ambientes de baixo recurso. }\end{array}$ \\
\hline $\begin{array}{l}\text { Ruan, D. } \\
2016\end{array}$ & $\begin{array}{l}\text { Int J Clin Exp } \\
\text { Med/SCOPUS }\end{array}$ & $\begin{array}{l}\text { Modified early warning } \\
\text { score in assessing disease } \\
\text { conditions and prognosis } \\
\text { of } 10,517 \text { pre-hospital } \\
\text { emergency cases }\end{array}$ & $\begin{array}{l}\text { Estudar os valores e a } \\
\text { viabilidade do MEWS na } \\
\text { avaliação da condição da } \\
\text { doença e previsão de } \\
\text { óbito em casos de } \\
\text { emergência pré- } \\
\text { hospitalar }\end{array}$ & $\begin{array}{l}\text { Estudo } \\
\text { prospectivo } \\
\text { com coleta do } \\
\text { MEWS em } \\
10.517 \\
\text { pacientes } \\
\text { (Evidência 1A) }\end{array}$ & $\begin{array}{l}\text { O MEWS possui certas } \\
\text { habilidades de distinção em } \\
\text { relação à avaliação da condição } \\
\text { da doença e previsão de morte e } \\
\text { poderia ser usado como uma } \\
\text { ferramenta eficaz para gerenciar } \\
\text { as emergências pré hospitalares. }\end{array}$ \\
\hline
\end{tabular}




\begin{tabular}{|c|c|c|c|c|c|}
\hline $\begin{array}{l}\text { Galen, L.V } \\
2016\end{array}$ & $\begin{array}{l}\text { PLOS } \\
\text { ONE/SCOPUS }\end{array}$ & $\begin{array}{l}\text { A Protocolised Once a } \\
\text { Day Modified Early } \\
\text { Warning Score (MEWS) } \\
\text { Measurement Is an } \\
\text { Appropriate Screening } \\
\text { Tool for Major Adverse } \\
\text { Events in a General } \\
\text { Hospital Population }\end{array}$ & $\begin{array}{l}\text { Analisar a aderência do } \\
\text { protocolo a um protocolo } \\
\text { MEWS na vida real para } \\
\text { determinar o valor } \\
\text { preditivo da medição } \\
\text { diária de MEWS nos } \\
\text { sérios eventos adversos: } \\
\text { morte, paradas cardíacas, } \\
\text { internações em UTI e } \\
\text { readmissões. }\end{array}$ & $\begin{array}{l}\text { Estudo } \\
\text { prospectivo } \\
\text { (Evidência 1A) }\end{array}$ & $\begin{array}{l}\text { A adesão ao protocolo foi alta, } \\
\text { embora um terço dos escores } \\
\text { críticos tenha sido calculado } \\
\text { errado. Pacientes com um MEWS } \\
3 \text { experimentaram } \\
\text { significativamente mais eventos } \\
\text { adversos. O valor preditivo } \\
\text { negativo de MEWS no início da } \\
\text { manhã <3 foi de } 98,1 \% \text {, } \\
\text { indicando a confiabilidade de essa } \\
\text { pontuação como uma ferramenta } \\
\text { de triagem. }\end{array}$ \\
\hline $\begin{array}{l}\text { Ssemmanda, } \\
\text { H } 2016\end{array}$ & $\begin{array}{l}\text { Critical Care } \\
\text { Research and } \\
\text { Practice/WEB } \\
\text { OF SCIENCE }\end{array}$ & $\begin{array}{l}\text { Abnormal Admission } \\
\text { Chest X-Ray and MEWS } \\
\text { as ICU Outcome } \\
\text { Predictors in a Sub- } \\
\text { Saharan Tertiary } \\
\text { Hospital: A Prospective } \\
\text { Observational Study }\end{array}$ & $\begin{array}{l}\text { Estudar radiografia de } \\
\text { admissão e MEWS como } \\
\text { preditores de mortalidade } \\
\text { de pacientes em UTI que } \\
\text { necessitam de ventilação } \\
\text { mecânica. }\end{array}$ & $\begin{array}{l}\text { Estudo } \\
\text { descritivo } \\
\text { prospectivo } \\
\text { (Evidência 1A) }\end{array}$ & $\begin{array}{l}\text { O trauma }(31 \%) \text { causou a maioria } \\
\text { das admissões na UTI, com } \\
\text { radiografia torácica de admissão } \\
\text { anormal associada positivamente } \\
\text { à mortalidade e um MEWS alto } \\
\text { também foram um bom preditor } \\
\text { de mortalidade. }\end{array}$ \\
\hline Kia, A 2020 & $\begin{array}{l}\text { J. Clin. } \\
\text { Med/WEB OF } \\
\text { SCIENCE }\end{array}$ & $\begin{array}{l}\text { MEWS++: Enhancing the } \\
\text { Prediction of Clinical } \\
\text { Deterioration in Admitted } \\
\text { Patients through a } \\
\text { Machine Learning Model }\end{array}$ & $\begin{array}{l}\text { Descrever um modelo de } \\
\text { aprendizado de uma } \\
\text { máquina chamada } \\
\text { MEWS ++ que permite a } \\
\text { identificação de pacientes } \\
\text { em risco de } \\
\text { encaminhamento de } \\
\text { atendimento ou morte } \\
\text { seis horas antes do } \\
\text { evento. }\end{array}$ & $\begin{array}{l}\text { Estudo } \\
\text { retrospectivo } \\
\text { de coorte } \\
\text { (evidência 2A) }\end{array}$ & $\begin{array}{l}\text { Este estudo constatou que o uso } \\
\text { de máquina e dados clínicos de } \\
\text { deterioração clínica ou óbito } \\
\text { podem ser previstos seis horas } \\
\text { antes do evento. O modelo que } \\
\text { desenvolveram pode alertar sobre } \\
\text { a deterioração do paciente horas } \\
\text { antes do evento, ajudando assim a } \\
\text { tomar decisões clínicas oportunas. }\end{array}$ \\
\hline Cakir, E 2019 & $\begin{array}{l}\text { Yoğun Bakım } \\
\text { Derg/WEB OF } \\
\text { SCIENCE }\end{array}$ & $\begin{array}{l}\text { Comparison of the } \\
\text { Effectiveness of Acute } \\
\text { Physiology and Chronic } \\
\text { Health Evaluation II and } \\
\text { Modified Early Warning } \\
\text { Score Scoring Systems in } \\
\text { Predicting Mortality in } \\
\text { Patients in the Intensive } \\
\text { Care Unit }\end{array}$ & $\begin{array}{l}\text { Avaliar a capacidade do } \\
\text { APACHE II e do MEWS } \\
\text { na previsão da } \\
\text { mortalidade de pacientes } \\
\text { internados em unidade de } \\
\text { terapia intensiva. }\end{array}$ & $\begin{array}{l}\text { Estudo } \\
\text { retrospectivo } \\
\text { (evidência 2A) }\end{array}$ & $\begin{array}{l}\text { Embora o MEWS tenha sido } \\
\text { superior ao escore APACHE II, } \\
\text { ambos os sistemas foram } \\
\text { significativamente eficaz na } \\
\text { previsão da mortalidade. }\end{array}$ \\
\hline $\begin{array}{l}\text { Ryan, H.M } \\
2017\end{array}$ & $\begin{array}{l}\text { J Obstet } \\
\text { Gynaecol/WE } \\
\text { B OF } \\
\text { SCIENCE }\end{array}$ & $\begin{array}{l}\text { Validating the } \\
\text { Performance of the } \\
\text { Modified Early Obstetric } \\
\text { Warning System } \\
\text { Multivariable Model to } \\
\text { Predict Maternal } \\
\text { Intensive Care Unit } \\
\text { Admission }\end{array}$ & $\begin{array}{l}\text { Avaliar o desempenho de } \\
\text { um Sistema Alerta } \\
\text { Obstétrico precoce } \\
\text { (MEOWS) para predizer } \\
\text { admissão em UTI } \\
\text { materna. }\end{array}$ & $\begin{array}{l}\text { Estudo de caso } \\
\text { controle } \\
\text { (evidência 3A) }\end{array}$ & $\begin{array}{l}\text { Alterar os parâmetros de disparo } \\
\text { do MEOWS pode melhorar a } \\
\text { precisão do MEOWS na previsão } \\
\text { da admissão na UTI. } \\
\text { É necessário um sistema de } \\
\text { pontuação do MEOWS para dar } \\
\text { suporte ao atendimento baseado } \\
\text { em evidências. }\end{array}$ \\
\hline $\begin{array}{l}\text { Roney, J.K } \\
2019\end{array}$ & $\begin{array}{l}\text { Nurs } \\
\text { Forum/WEB } \\
\text { OF SCIENCE }\end{array}$ & $\begin{array}{l}\text { Implementation of a } \\
\text { MEWS-Sepsis screening } \\
\text { tool: Transformational } \\
\text { outcomes of a nurse-led } \\
\text { evidence-based practice } \\
\text { project }\end{array}$ & $\begin{array}{l}\text { Avaliar, revisar e } \\
\text { implementar uma } \\
\text { ferramenta de triagem } \\
\text { MEWS - Sepse } \\
\text { modificada em } \\
\text { tratamento intensivo. }\end{array}$ & $\begin{array}{l}\text { Ciclo de } \\
\text { planejamento, } \\
\text { cenário } \\
\text { simulado, } \\
\text { coleta de dados } \\
\text { de pacientes } \\
\text { com sépticos } \\
\text { (evidência 2C) }\end{array}$ & $\begin{array}{l}\text { A implementação de uma } \\
\text { ferramenta de triagem MEWS - } \\
\text { Sepse contribuiu para } \\
\text { identificação e implementação } \\
\text { precoces de intervenções } \\
\text { sensíveis ao tempo, destinadas à } \\
\text { prevenção de mortes associadas à } \\
\text { sepse. }\end{array}$ \\
\hline
\end{tabular}

Fonte: Autores (2020). 


\section{Discussão}

Demonstrou-se que a deterioração da gravidade clínica no ambiente hospitalar exige a adoção de intervenções efetivas e resolutivas. Revelou-se ainda que nas unidades de Pronto Socorro e UTI os escores preditores de gravidade organizados em sistemas de pontuação auxiliam a tomada de decisões e a determinação da gravidade da doença (Akgun, Ertan \& Yucel, 2018).

Apontou-se que o MEWS pode ser eficaz na identificação precoce da deterioração clínica em várias situações em que a deterioração clínica se instala, em maior ou menor severidade. Nota-se que quando realizado na admissão do doente no serviço de urgência ou diariamente na enfermaria, mostra-se como um bom preditor do risco de mortalidade intra-hospitalar, e da necessidade de admissão em UTI (Alexandre, Gomez, Marques, Nunes \& Gomes, 2019). Ressalta-se que um MEWS classificado acima de 3 é um forte preditor de desfechos clínicos como mortalidade hospitalar, readmissões na UTI em até 30 dias e o prolongamento do tempo de internação. Identificou-se além disso, o valor preditivo de MEWS <3 na população hospitalar geral foi de 98,1\%, indicando a confiabilidade desse escore como ferramenta de triagem (Galen, Dijkstra, Ludikhuize, Krame \& Nanayakkara, 2016).

Infere-se que a avaliação clínica realizada pelo enfermeiro precisa estar amparada em parâmetros objetivos e na formulação de escores cuja aplicação seja viável para toda equipe profissional. A instituição de uma busca ativa por pacientes com evidência de instabilidade clínica e deterioração hemodinâmica, e a sua avaliação por meio do MEWS pode impactar na ocorrência de eventos graves, na redução do risco de morte e na instituição de intervenções eficazes e oportunas (Stafseth, Grobeck, Lien, Randen \& Lerdal, 2016). Enfatiza-se que o valor de corte do MEWS (para previsão de mortalidade hospitalar relatada em estudos) possui uma variação de acordo com a população e locais de pesquisa. Explicitando-se a necessidade de validação do MEWS em cada instituição a fim de delimitar o melhor ponto de corte, que obtenha maior sensibilidade e especificidade, de acordo com as necessidades dos pacientes no referido contexto, a disposição de recursos tecnológicos e humanos e a presença ou não de um Time de Resposta Rápida (TRR). Ratifica-se que os resultados dos estudos expostos acima nos mostram a eficácia do MEWS no que este escore se propõe a fazer, dentro da realidade de cada serviço hospitalar, levando em consideração também, o perfil dos pacientes assistidos.

Realizou-se uma comparação entre escores de alerta precoce para predizer a motalidade dos pacientes críticos acometidos pelo coronavírus. Foram selecionados 138 pacientes gravemente enfermos e os escores utilizados foram o MEWS e o Rapid Emergency Medicine Score (REMS). Revelou-se que o REMS tem uma sensibilidade para previsão de mortalidade uma vez que no seu valor de corte ideal (> ou igual a 6) tem sensibilidade de $89,5 \%$ e uma especificidade de $69,8 \%$. Salientase, por outro lado, que o REMS inclui mais 2 variáveis, sendo: idade e saturação de oxigênio (Hu, Yao \& Qui, 2020).

Constatou-se em um estudo, em alas cirúrgicas realizado num período de 12 meses e contou com 3827 pacientes em duas enfermarias cirúrgicas, mostrou que os TRR eram chamados principalmente por complicações cardiopulmonares, com destaque para a parada cardíaca. Identificou-se que os gatilhos para a ativação do TRR foram alertas anormais de ECG (assistolia e AESP).

Implantou-se o MEWS foi através de um sistema automatizado e, deste modo, o número de notificações e a disponibilidade de dados sobre funções vitais na chegada do TRR eram significativamente maiores. Destaca-se que quando o paciente apresentava MEWS pontuado entre 1 a 4 a enfermeira responsável era informada e agendava a próxima verificação para as próximas 4 a 6 horas. Se o MEWS era pontuado entre 5 a 6 , o médico da enfermaria e a enfermeira do setor eram informados. Estabelece-se como ponto de corte 7 como critério para se identificar a deterioração clínica e ativação do TRR. A taxa de paradas cardíacas caiu significativamente de 5,3 para 2,1 por 1.000 admissões no período de intervenção $(\mathrm{P}<0,001)$. Destaca-se que essa observação foi paralela à redução de internações não planejadas na UTI de 3,6\% para 3,0\% $(\mathrm{P}<0,001)$ (Heller, Mees, Lauterwald, Reeps, Koch \& Weitz, 2020). 
Identificou-se um estudo em que foram sugeridas avaliações complementares ao MEWS tais como as radiografias de tórax e no cenário do estudo a identificação de uma radiografia torácica com achados anormais na admissão do paciente passou a ser usado como indicador para se prever a mortalidade entre pacientes internados na UTI; no entanto, essa previsão não foi conclusiva, dada sua baixa especificidade. Identificou-se também que um MEWS $\geq 5$ é um eficaz preditor de mortalidade em pacientes internados em UTI usando Ventilação Mecânica Invasiva (VMI). Revelou-se que os dois métodos em conjunto podem servir como preditores da necessidade de intervenções que salvam vidas precocemente e diminuem a mortalidade em UTI (Ssemmanda, Kuggya, Lubulwa, Muvibda, Kwitonda \& Wanzira, 2016).

Enfatiza-se que o escore de alerta precoce também já foi adaptado outras vezes às necessidades da equipe profissional de acordo com a realidade do local de aplicação do escore. Por exemplo, para avaliar pacientes com malignidade hematológica foi adicionado a variável de razão entre SpO2 / FiO2 (MEWS-SF) para aumentar a especificidade do MEWS. O valor de corte para predizer a admissão na UTI usando o MEWS foi de 6 (sensibilidade: 77,7\%, especificidade: 74,4\%, valor preditivo positivo: 72,7\%, valor preditivo negativo: 79,1\%); já o valor de corte pelo escore MEWS-SF foi de 7 (sensibilidade: 83,5\%, especificidade: $80,3 \%$, valor preditivo positivo: 79,0\%, valor preditivo negativo: 84,7\%). Observou-se, diante dos dados, que o MEWS-SF forneceu uma avaliação aprimorada para se prever a admissão na UTI e a mortalidade hospitalar, em comparação com o MEWS tradicional neste grupo de pacientes (Lee, Jung, Kim, Koh, Lim \& Honh, 2020).

Realizou-se, em outro cenário, a adaptação do MEWS com inclusão de critérios para se realizar a triagem para a sepse em UTI. Considera-se a sepse como um grave problema de saúde pública e com base em recente revisão sistemática, a sua incidência mundial é estimada em 31,5 milhões de casos por ano, com potencial de acarretar 5,3 milhões de óbitos (Fleischmann, Scherag, Adhikari, Hartog, Tsaganos, Schlattmann, 2016). Identificou-se no estudo SPREAD (Sepsis PREvalence Assessment Database), um levantamento nacional da prevalência em 1 único dia em UTIs brasileiras a incidência para a sepse de 36,3 casos por mil pacientes-dias e uma mortalidade hospitalar em torno de 55,7\% (Machado, Cavalcanti, Bozza, Ferreira, Carrara \& Sousa, 2017). Aponta-se em outro estudo os componentes de triagem de sepse para temperatura, frequência respiratória e frequência cardíaca foram ajustados para corresponder aos parâmetros da Síndrome da Resposta Inflamatória Sistêmica (SIRS), a saturação de oxigênio $\left(\mathrm{SPO}_{2}\right)$ foi alterada para "taxa de fluxo de oxigênio", e os valores de ácido lático e contagem de glóbulos brancos foram adicionados ao MEWS. Constatou-se que a taxa de mortalidade por sepse observada diminuiu de $17 \%$ - 25\% a $11 \%$ - 18\%; após um ano da implementação dessa ferramenta MEWS-SEPSE, a organização percebeu um declínio na mortalidade por sepse de 24\% (Roneym Whithey \& Longa, 2020).

Considera-se que uma das estratégias conhecidas para aprimorar o gerenciamento da assistência ao paciente com sepse é a identificação precoce dos pacientes que possuem risco mais elevado de desenvolver eventos adversos. Comprovou-se como resultado do estudo que esta identificação pode ajudar a equipe multiprofissional a iniciar um tratamento precoce com antibioticoterapia adequada, ressuscitação volêmica, entre outras intervenções. Revela-se ainda que os escores de alerta precoce (SOFA e qSOFA) mais específicos para os pacientes com sepse foram desenvolvidos com o propósito de se investigar sinais de falência orgânica.

Evideciou-se em estudo retrospectivo com 1.589 pacientes comparou a precisão do MEWS, National Early Warning Score (NEWS) e do Search Out Severity (SOS) com o Sequential Organ Failure Assessment (SOFA) e do Quick SOFA (qSOFA) para predizer resultados em pacientes com sepse. Revela-se que escore SOFA forneceu uma especificidade mais alta para se prever a mortalidade hospitalar em 30 dias, seguido por SOS, MEWS, qSOFA e foi mais baixo para o NEWS. Usando o valor médio dos pacientes sobreviventes, o SOFA com valor 7 apresentou sensibilidade de 95,3\%, especificidade de 49,4\%, o SOS com valor 8 apresentou uma sensibilidade de $94,9 \%$ e especificidade de 44,5\%, para mortalidade hospitalar. Para prever a mortalidade na UTI e melhor precisão para predizer a falência de múltiplos órgãos, o escore SOFA é o que possui melhor especificidade, seguido do SOS, posteriormente aparece o MEWS, o qSOFA e, por fim o NEWS com uma sensibilidade 
menor. Identificou-se neste mesmo estudo com pacientes com diagnóstico de sepse, que o escore SOFA teve a melhor precisão para predizer a falência de múltiplos órgãos, bem como a curva ROC do SOFA e do SOS foram estatisticamente superiores aos outros escores apresentados (Khwannimit, Bhurayanontachai \& Vattanavanit, 2019). Observa-se que quanto mais específica for a adaptação do escore para o perfil do paciente melhor serão a sua especificidade e sensibilidade. Revelando-se que a modificação do MEWS baseada na caracterização dos pacientes em risco melhora a sensibilidade e especificidade dos pontos de corte fornecendo valores mais assertivos (Lee et al., 2020).

Observou-se que quando falamos em eventos traumáticos, o MEWS pode ser usado no Atendimento Pré-Hospitalar objetivando a detecção de pacientes que necessitem de uma assistência com maior complexidade. Identificou-se em um estudo que o valor do MEWS pré-transferência estava associado a maus resultados clínicos, ao modo de transporte e à gravidade da lesão. Ressalta-se que em 587 pacientes o MEWS pré-transferência mostrou uma relação linear significativa com a mortalidade, admissão na UTI, transporte aéreo e lesões graves. Destaca-se que o valor do MEWS no momento da prétransferência também ajudou a prever um procedimento cirúrgico. No cenário de Atendimento Pré-Hospitalar, o profissional pode aplicar o MEWS como uma medida que pode diminuir a chance de subestimar um desfecho clínico desfavorável e assim postergar a transferência de um paciente (Salottolo, Carrick, Johnson, Gamber \& Bar-Or, 2020).

Pontua-se que na Etiópia, uma instituição buscou implementar o MEWS em pacientes obstétricas como preditor de admissão na UTI onde foi realizado um estudo retrospectivo e de caso-controle onde os resultados mostraram que o modelo MEWS obstétrico foi altamente sensível à previsão de admissão na UTI. Acredita-se, porém, mulheres sem complicações clínicas e com MEWS classificado como gatilho verde também podem desenvolver complicações e apresentarem um MEWS gatilho vermelho (Ryan, Jones, Payne, Sharma, Hutfield \& Lee, 2017).

Revela-se que a mortalidade materna tem sido representada como a "ponta do iceberg", em que sua base corresponde à morbidade total por problemas de saúde ligados ao ciclo gravídico-puerperal (Firoz, Chou, Dadelszen, Agrawal, Vanderkruik \& Tuncalp, 2013). Um estudo descritivo realizado com 705 mulheres internadas que buscou avaliar o MEWS obstétrico após a gestação detectou a existência de eventos gatilho em $49,8 \%$ das pacientes; porém em apenas três casos $(0,82 \%)$ o médico foi chamado para atendimento. Observou-se ainda que 26,8\% das pacientes apresentaram mais de um gatilho vermelho e $67,0 \%$ delas apresentaram mais de dois alertas amarelos durante o período de internamento. Considerando-se o alerta vermelho como pressão arterial sistólica ou diastólica elevada, foi verificado que esse gatilho esteve presente em aproximadamente 19\% das pacientes. Acrescenta-se ainda que $83,3 \%$ das pacientes foram classificadas como de alto risco obstétrico e que $49,8 \%$ delas apresentaram anormalidades nos parâmetros fisiológicos, demonstrado pela identificação dos eventos gatilho no Escore de Alerta Precoce Modificado Obstétrico MEOWS (Schuler, Katz, Melo \& Coutinho, 2019).

Pontua-se como outra conclusão deste estudo, o fato de que programas de educação continuada precisam ser oferecidos aos profissionais envolvidos na assistência obstétrica para que estes articulem o significado das alterações nos sinais vitais com as ações necessárias de acordo com os gatilhos.

Sabe-se que os escores de alerta precoce, em sua maioria, aparecem, também, como instrumentos de cuidado com relação à cultura de Segurança do Paciente. Infere-se assim que a segurança do paciente constitui um dos desafios para as instituições que prestam cuidados em saúde do século XXI. É foco de discussão, tanto em âmbito nacional quanto internacional, dada sua importância para o sistema de saúde e as repercussões para a sociedade em geral. Os profissionais de saúde têm dificuldades de manter uma comunicação que favoreça o trabalho em equipe e a continuidade da assistência intra e extra-hospitalar, seja por falta de tempo, escassez de pessoal, ausência de padronização, imperícia ou desconhecimento da importância de tal ação (Olino, Gonçalves, Strada, Vieira, Machado \& Molina. 2019. 


\section{Conclusão}

Contatou-se que o MEWS tem sido utilizado pelo enfermeiro na prática clínica hospitalar nas seguintes situações: prever eventos adversos de pior prognóstico clínico como a PCR, óbitos e (re)admissão na UTI; no processo gerencial das unidades de internação, fazendo com que a assistência seja efetiva de acordo com o grau de criticidade do paciente que é atendido no âmbito hospitalar e na melhora da comunicação entre a equipe. Revelou-se, neste aspecto, que há evidências do aumento da eficácia na tomada de decisões entre a equipe multiprofissional, visando uma maior atenção para os sinais de deterioração clínica e, consequentemente, uma intervenção segura e imediata.

Discute-se bastante acerca da versatilidade do escore de alerta precoce uma vez que a sua aplicação no ambiente hospitalar é variada abrangendo diversos perfis clínicos. Deve-se atentar para a escassez de estudos acerca do MEWS no Brasil, o que nos leva a buscar conhecer melhor a realidade estrangeira do seu uso. Fazem-se necessários outros estudos sobre os escores de alerta precoce em realidades diferentes do Brasil, objetivando uma aplicação de maneira eficaz como ferramenta preditora de piores desfechos clínicos; ouro aspecto as ser destacado é o fato de que ele pode se constituir como um instrumento que favoreça a autonomia profissional do enfermeiro.

Ressalta-se que este trabalho visa contribuir para um avanço e crescimento da assistência de enfermagem e da equipe multiprofissional no que diz respeito ao cuidado ao paciente crítico e ao paciente que mostra sinais de deterioração clínica. Esta revisão abordou múltiplas facetas do MEWS, bem como aplicações de outros escores de alerta precoce. Deste modo, a contribuição pode ser vista, também, no escopo do aumento da literatura disponível para embasamento teórico para a prática clínica, uma vez que a quantidade de estudos relacionando a atuação do enfermeiro com a utilização dos escores de alerta precoce são escassos.

Para futuros trabalhos sugere-se que haja um aprofundamento acerca do uso de escores de alerta precoce e sua implicação na melhoria da assistência de enfermagem, de pacientes críticos ou não, uma vez que a literatura já relata de maneira positiva e afetiva a sua contribuição para melhores indicadores de performance em saúde. No Brasil possuímos poucos estudos que possam dar uma base científica forte para que as ações de saúde sejam pautadas. Deste modo, estudos futuros podem elucidar dúvidas e questões que possam aparecer frente ao processo de criação e implementação de um escore de alerta precoce nos serviços de saúde.

\section{Referências}

Akgun, F. S., Ertan, C., Yucel, N. (2018). The prognastic efficiencies of modified early warning score and mainz emergency evaluation score for emergency department patients. Niger J Clin Pract, 21(12), 1590-5.

Alexandre, A. R., Gomez, C., Marques, A., Nunes, A., Gomes, J. A. (2019). Modified Early Warning Score como preditor de readmissões precoces numa unidade de cuidados intensivos: um estudo de caso-controlo. Med interna (Caracas), 26(3), 1-6.

Bekhit, O. E. S. M., Algameel, A. A., Eldash, H. H. (2014). Application of pediatric index of mortality version 2: score in pediatric intensive care unit in an African developing country. Pan African Medical Journal, 17, 1-6.

Botelho, L. L. R., Cunha, C. C. A., Macedo, M. (2011). O método da revisão integrativa nos estudos organizacionais. Revista eletrônica gestão e sociedade, $5(11), 1-16$

Brasil Ministério da Saúde. (2020). Orientações para manejo de pacientes com COVID-19. Brasília, DF: MS.

Cipriano, E. S. V., Salgado, B. S., Oliveira, N. A., Aguiar, B. G. C. (2018). Implantação do Score de deterioração clínica (MEWS) em um hospital privado da cidade do Rio de Janeiro e seus respectivos resultados. Enfermagem Brasil [Internet], 1 -12.

Firoz, T., Chou, D., Dadelszen, P. V., Agrawal, P., Vanderkruik, R., Tunçalp, O., et al. (2013). Measuring maternal health: focus on maternal morbidity. Bull World Health Organ, 91(10): 794-6.

Fleischmann, C., Scherag, A., Adhikari, N. K. J., Hartog, C. S., Tsaganos, T., Schlattmann, P., et al. (2016). Assessment of Global Incidence and Mortality of Hospital-treated Sepsis. Current Estimates and Limitations. Am J Respir Crit Care Med, 193(3): 259-72.

Galen, L. S. V., Dijkstra, C. C., Ludikhuize, J., Krame, M. H. H., Nanayakkara, P. W. B. (2016). A Protocolised Once a Day Modified Early Warning Score (MEWS) Measurement Is an Appropriate Screening Tool for Major Adverse Events in a General Hospital Population. PLoS One [Internet]. 11(8), 1-11. 
Heller, A. R., Mees, S. T., Lauterwald, B., Reeps, C., Koch, T., Weitz, J. (2020). Detection of Deteriorating Patients on Surgical Wards Outside the ICU by an Automated MEWS-Based Early Warning System With Paging Functionality. Ann Surg, 271(1), 100-5.

Hu, H.; Yao, N.; Qiu, Y. (2020). Comparing Rapid Scoring Systems in Mortality Prediction of Critically Ill Patients With Novel Coronavirus Disease. Medicina Academica de Emergência, 27(6), 461-8.

Khwannimit, B., Bhurayanontachai, R., Vattanavanit, V. (2019). Comparison of the accuracy of three early warning scores with SOFA score for predicting mortality in adult sepsis and septic shock patients admitted to intensive care unit. Heart Lung, 48(3): 240-4.

Lee, J. R., Jung, Y. K., Kim, H. J., Koh, Y., Lim, C. M., Honh, S. B., et al. (2020). Derivation and validation of modified early warning score plus SpO2/FiO2 score for predicting acute deterioration of patients with hematological malignancies. Korean J Intern Med. 1-14.

Machado, F. R., Cavalcanti, A. B., Bozza, F. A., Ferreira, E. M., Carrara, F. S. A., Sousa, J. L., et al. (2017). The epidemiology of sepsis in Brazilian intensive care units (the Sepsis PREvalence Assessment Database, SPREAD): an observational study. Lancet Infect Dis, 17(11): 1180-9.

Montenegro, S. M. S. L. (). Avaliação do desempenho de alerta precoce modificado em hospital público brasileiro [dissertação]. Ribeirão Preto: Faculdade de Medicina de Ribeirão Preto da Universidade de São Paulo. Programa de Pós-Graduação em Mestrado Profissionalizante em Gestão de Organizações de Saúde.

Morales, C. L. P. (2016). Avaliação de pacientes graves em emergência e terapia intensiva a partir da escala MEWS: revisão sistemática sem metanálise [dissertação]. Florianópolis: Universidade Federal de Santa Catarina. Programa de Pós-Graduação em Enfermagem.

Noronha, K. V. M. S., Guedes, G. R., Turra, C. M., Andrade, M. V., Botega, L., Nogueira, D. (2020). Pandemia por COVID-19 no Brasil: análise da demanda e da oferta de leitos hospitalares e equipamentos de ventilação assistida segundo diferentes cenários. Cad. Saúde Pública. 36(6), 1-17.

Olino, L., Gonçalves, A. C., Strada, J. K. R., Vieira, L. B., Machado, M. L. P., Molina, K. L., et al. (2019). Comunicação efetiva para a segurança do paciente: nota de transferência e Modified Early Warning Score. Rev gaúch enferm, 40(no.spe), 1-6.

Rocha, T. F., Neves, J. G., Viegas, K. (2016). Escore de alerta precoce modificado: avaliação de pacientes traumáticos. Revista Brasileira de Enfermagem, 69(5), 1-5.

Roney, J. K., Whithey, B.E., Longa, J. D. (2020). Implementation of a MEWS-Sepsis screening tool: Transformational outcomes of a nurse-led evidencebased practice project. Nurs Forum, 55(2): 144-8.

Ryan, H. M., Jones, A. U., Payne, B. A., Sharma, S., Hutfield, A. M., Lee, T., et al. (2017). Validating the Performance of the Modified Early Obstetric Warning System Multivariable Model to Predict Maternal Intensive Care Unit Admission. J Obstet Gynaecol Can, 39(9): 728-33.

Salottolo, K., Carrick, M., Johnson, J., Gamber, M., Bar-Or, D. (2017). A retrospective cohort study of the utility of the modified early warning score for interfacility transfer of patients with traumatic injury. BMJ Open, 7(5), 1-6.

Schuler, L., Katz, L., Melo, B. C. P., Coutinho, I. C. (2019). Aplicação do Modified Early Obstetric Warning System (MEOWS) em mulheres após gestações: um estudo descritivo. Rev. Bras. Saude Mater. Infant, 19(3): 545-55.

Ssemmanda, H., Luggya, T. S., Lubulwa, C., Muyinda, Z., Kwitonda, P., Wanzira, H. (2016). Abnormal Admission Chest X-Ray and MEWS as ICU Outcome Predictors in a Sub-Saharan Tertiary Hospital: A Prospective Observational Study. Crit Care, 1-7.

Stafseth. S. K., Grønbeck, S., Lien, T., Randen I, Lerdal A. The experiences of nurses implementing the Modified Early Warning Score and a 24-hour on-call Mobile Intensive Care Nurse: An exploratory study. Intensive crit care nurs, 34, 25-33.

UTIs Brasileiras Registro Nacional de Terapia Intensiva [homepage na internet]. AMIB. (2020). Principais desfechos nas UTIs brasileiras.

World Health Organization. (2020). Oxygen sources and distribution for COVID-19 treatment centres: interim guidance. 2020. 1-6. 